\title{
Effects of Intra-vitreal Injection of Bevacizumab as an Adjunct during Phacoemulsification in Diabetic Maculopathy
}

\author{
Ali Afzal Bodla ${ }^{1}$, Syeda Minahil Kazmi ${ }^{2}$, Noor Tariq ${ }^{3}$, Ayeza Moazzam ${ }^{4}$, Muhammad Muneeb Aman ${ }^{5}$ \\ ${ }^{i-5}$ Department of Ophthalmology, Multan Medical and Dental College, Multan
}

\begin{abstract}
Purpose: To study the effects of Intra-vitreal injection of Bevacizumab as an adjunct during phacoemulsification in patients with diabetic retinopathy.
\end{abstract}

Study Design: Quasi experimental study.

Place and Duration of Study: Multan Medical and Dental College and Bodla Eye Care, Multan, between March 2018 to February 2019.

Methods: The study included 108 eyes of 108 diabetic patients who were scheduled to undergo phacoemulsification. They were equally divided into two groups; Bevacizumab and control group. Complete ocular examination and macular thickness and volume were determined using an OPTOVUE-OCT machine. The patients in the Bevacizumab group were given intra-vitreal injection of $1.25 \mathrm{mg} / 0.05 \mathrm{ml}$ of Bevacizumab at the time of Phacoemulsification.

Results: The bevacizumab group manifested low value of central macular thickness (CMT) one-month postsurgery as compared to the control group (262.2 \pm 32.2 and $288.5 \pm 54.1$, respectively) with $P=0.01$. The Total macular volume, and Best-corrected visual acuity in the two groups showed no significant difference one month after surgery. Two (4\%) patients in the Bevacizumab group and five (10\%) patients in the control group developed post-surgical macular edema one month after surgery which was not statistically significant $(P=0.244)$. We found no significant relationship between the post-surgical macula edema with the presence of mild Non Proliferative Diabetic Retinopathy. (Fisher's test, $P=0.321$ ).

Conclusion: Ocular anti-VEGF therapy substantially reduces macular edema secondary to post-surgical inflammation in diabetic patients. It effectively reduces the central macular thickness although the results are not found to be statistically significant when compared with the control group.

Key Words: Diabetes mellitus; diabetic macular edema; diabetic retinopathy, Bevacizumab.

How to Cite this Article: Bodla AA, Kazmi SM, Tariq N, Moazzam A, Aman MM. Effects of Intra-vitreal Injection of Bevacizumab as an Adjunct during Phacoemulsification in Diabetic Maculopathy. Pak J Ophthalmol. 2021, 37 (1): $7-11$.

Doi: https://doi.org/10.36351/pjo.v37i1.1122

Correspondence: Syeda Minahil Kazmi

Department of Ophthalmology

Multan Medical and Dental College

Multan

Email: minahilkazmi257@gmail.com

Received: August 21, 2020

Accepted: October 22, 2020

\section{INTRODUCTION}

Subsequent to cataract surgery performed on diabetics, the most sight threatening retinopathy is gross and/or cystoid macular edema, which is indicated as fluid accumulation in the macula. ${ }^{1-3}$ After cataract surgery there is a substantial increment in angiogenesis and inflammation, thereby surging cytokines, chemokines and vaso-permeability factors in the vascular 
endothelium which trigger breakdown of blood retinal barrier, manifesting clinically as macular edema and resulting in visual loss. ${ }^{4,5}$ The pathophysiology encompasses between the vascular endothelial growth factor (VEGF) and inflammatory mediators. ${ }^{6,7}$ This study has been conducted to clinically investigate the potential therapeutic effects of Anti VEGFs in the control of macular edema in patients with nonproliferative diabetic retinopathy.

\section{METHODS}

In this study, we enrolled 108 eyes of 108 patients from hospital of Multan Medical and Dental College and Bodla Eye Care, Multan, between March 2018 to February 2019. A written Ethical Review statement was obtained before the beginning of study and the research was conducted according to the principles of Declaration of Helsinki. These patients had significant cataract but with visible posterior segment. At the first visit patients were divided into control (got phacoemulsification alone) and intervention group (who got intravitreal injection of $1.25 \mathrm{mg} / 0.05 \mathrm{ml}$ of Bevacizumab at the end of surgery) depending upon their treatment choice. The inclusion criteria was diabetic patients (Diabetes Mellitus Type 2) with significant cataract and early diabetic retinopathy or moderate Non Proliferative Diabetic Retinopathy. The exclusion criteria were patients with preoperative central macular thickness (CMT) of $>280 \mu \mathrm{m}$ and/or any attestation of cystic spaces indicated by optical coherence tomography (optovue-OCT; OPTOVUE companies; USA), HbA1c greater than 6.8, intractable Non Proliferative Diabetic Retinopathy or Proliferative Diabetic Retinopathy, Diabetes Mellitus type 1, Uncontrolled persistent hypertension and Precedent retinal laser therapy. During the preliminary visit, the anterior and posterior segment examination was done using Slit lamp and 90 D lens. Goldmann Applanation tonometry was performed to determine the Intra Ocular pressure whereas OCT was used to assess retinal structures and macular thickness. ETDRS was employed to assess the degree of retinopathy. The complete post-operative ophthalmic examinations were performed at day one, day seven, and one month later. OCT was performed one month post-surgery on all patients.

By using the $512 \times 128$ scan pattern, OCT images were acquired and center of $6 \times 6-\mathrm{mm}$ scanning area was fixed at the fovea centralis. Central macular thickness (CMT) was measured which is the average thickness of retina in the central foveal $1 \mathrm{~mm}$ subfield. Total macular volume (TMV) was also determined. Phacoemulsification was performed by a single surgeon (Infinity ${ }^{\circledR}$ vision system; Alcon Laboratories). At the end of cataract surgery, an injection of $1.25 \mathrm{mg}$ of Bevacizumab was injected intravitreally given in the inferotemporal quadrant, $3.5 \mathrm{~mm}$ away from limbus using a standard $1 \mathrm{cc}$ insulin syringe.

It was pre-defined that CMT $>280 \mu \mathrm{m}$ using OCT would be considered as a consequential postoperative macular edema. SPSS version 20 was employed to analyze the statistical data. The Variables found in the data were demonstrated in terms of mean \pm standard error of mean. The analysis was done by using Wilcoxon Mann - Whitney test. It was determined that $P$ value less than 0.05 will be significant.

\section{RESULTS}

The study included 108 eyes from 108 patients. Three patients were omitted due to advancement of postoperative cystoid macular edema not secondary to diabetes as in accordance with guidelines suggested by Munk et al. Five of these patients were lost during follow up. After excluding the patients who were lost during follow-up, there were fifty eyes included in the Bevacizumab group and fifty eyes in the control group. The pre requisite criterion for the patients was Type $2 \mathrm{DM}$. The two groups were much the same in terms of age, male to female ratio, stage of diabetic retinopathy baseline CMT and TMV, HbAlc and blood pressure. The bevacizumab group manifested low value of CMT one-month post-surgery as compared to the control group (262.2 \pm 32.2 and 288.5 \pm 54.1 , respectively) with $P=0.02$. The TMV and BCVA in the two groups showed no significant difference one month after surgery (Table 1). In the light of our definition in study set in accordance with DRCR. NET protocols, (CMT > $280 \mu \mathrm{m}$ using SDOCT), two (4\%) patients in the Bevacizumab group and five $(10 \%)$ patients in the control group developed post-surgical macular edema one month after surgery. Although the frequency of macular edema was somewhat higher in the control group, this distinction was not statistically significant at one month after surgery $(P=0.244)$. We found no significant relationship between the post-surgical macula edema with the presence of mild Non Proliferative Diabetic Retinopathy. (Fisher's test, $P=0.321$ ). 
Table 1: Mean Central macular thickness (CMT) and total macular volume (TMV) before and at 1 month after cataract. CMT: Central Macular Thickness; TMV: Total Macular Volume; BCVA: Best Corrected Visual Acquity; PME: Postoperative macular edema.

\begin{tabular}{lccc}
\hline & Bevacizumab Group & Control Group & P-value \\
\hline CMT (pre-operative) & $255.4 \pm 24.3$ & $262.5 \pm 27.4$ & 0.30 \\
CMT (1 month post- operative) & $262.2 \pm 32.2$ & $288.5 \pm 54.1$ & 0.01 \\
TMV (pre operative) & $8.29 \pm 0.54$ & $8.30 \pm 0.51$ & 0.93 \\
TMV (1 month post-operative) & $8.30 \pm 0.62$ & $8.53 \pm 0.70$ & 0.14 \\
BCVA & $0.58 \pm 0.21$ & $0.50 \pm 0.09$ & 0.13 \\
BCVA (1 month post operative) & $0.08 \pm 0.06$ & $0.11 \pm 0.09$ & 0.226 \\
Post operative PME\% & $2(\% 4)$ & $5(\% 10)$ & 0.24 \\
\hline
\end{tabular}

\section{DISCUSSION}

Vascular endothelial growth factor is one of the key agents responsible for causing macular edema. AntiVEGF act as promising halters of this macular edema. In such patients a mono-therapy or combination of topical NSAIDs and corticosteroids can be employed right after the surgery as a prophylactic therapy or as treatment. ${ }^{8,9}$ However, there are other options which include intravitreal anti-VEGF and steroid therapy at the time of surgery. ${ }^{10,11}$ Multiple anti-VEGF drugs e.g. Afilibercept, Intravitreal Ranibizumab (IVR) or intravitreal Bevacizumab (IVB) are administered as an adjunct in cataract surgery for the management of macular edema in diabetic patients having danger of post-operative macular edema. ${ }^{12-16}$ These drugs are used widely and are effective in vision improvement and prevention of vision loss caused by diabetic macular edema. The effect of anti-VEGF is not enough sometimes and many patients do not show complete remission of fluid.

The optical coherence tomography (OCT) is used for the clinical assessment of diabetic macular edema that discerns visual loss by objective evaluation of macular thickness and evaluates the vitreo-macular interface. ${ }^{17,18}$ The most certain rationale of poor vision in diabetics after cataract surgery is Cystoid and/or gross macular edema. It has been demonstrated that aqueous VEGF levels are greatly responsible for clinically remarkable changes in Central Macular Thickness. The pre-operative VEGF levels play a pivotal role in causing post-operative macular edema. ${ }^{19}$ Therefore, it is hypothesized that controlling this VEGF would productively play a crucial role in impeding postoperative elevation in central macular thickness and thereby refining the visual outcomes in the patients. The present study showed that patients who had no or mild diabetic retinopathy had an insignificant decrease in macular thickness subsequently after the administration of intravitreal Bevacizumab injection at the time of surgery.

Takamura et al. appraised the effects of intravitreal Bevacizumab supplemented shortly after phacoemulsification in diabetics with pre-existing macular edema. ${ }^{12}$ While in first month post-surgery macular edema decreased proficiently by Bevacizumab, it somehow was increased in the control group. Likewise, best corrected visual acuity (BCVA) was significantly lower in the control group then in the bevacizumab group. Conducive to the hypothesis stating that total Macular Volume is a better reflection of changes in macular status rather than Central Macular Thickness, we measured both TMV and CMT in order to observe any change with the use of intravitreal Bevacizumab.

Often quoted, post-op macular edema was taken as an increase of CMT $\geq 60 \mu \mathrm{m}$ in relation to the preoperative baseline. Interestingly, CMT changes until 1 month after surgery were depicted differently in these two groups. Fard et al. in their study, found a significant increase in the control group one month post-operative as compared to the Bevacizumab group while evaluating the effect of intravitreal Bevacizumab in patients with CMT $<200 \mu \mathrm{m}$. They concluded that intravitreal administration of $1.25 \mathrm{mg}$ bevacizumab was effective in reducing macular thickness. ${ }^{20}$

It is apparent that progression of diabetic retinopathy or development of subsequent macular edema after surgery occurs more commonly in patients with moderate to severe NPDR and/or macular edema prior to phacoemulsification as compared to patients with no or mild NPDR. ${ }^{21}$

There are a few limitations to our study. First, sample size of each group was small and perhaps not considerable enough to explicate the beguiling differences particularly of post-operative cystoid 
macular edema between the control and the Bevacizumab group. Second, the follow up period was small. It is possible that some change would be imminent in further follow-ups. Third, macular edema was defined as CMT > $280 \mu \mathrm{m}$ using SD-OCT. A comparable conclusion might have been drawn by considering an increase of $\geq 60 \mu \mathrm{m}$.

\section{CONCLUSION}

The ocular anti-VEGF therapy substantially reduces central macular thickness secondary to post-surgical inflammation in diabetic patients. However, the results are not found to be statistically significant when compared with the control group.

\section{Ethical Approval}

The study was approved by the Institutional review board/ Ethical review board. (MMDC/B-24-418)

\section{Conflict of Interest}

Authors declared no conflict of interest

\section{REFERENCES}

1. Zhao LQ, Cheng JW. A Systematic Review and MetaAnalysis of Clinical Outcomes of Intravitreal AntiVEGF Agent Treatment Immediately after Cataract Surgery for Patients with Diabetic Retinopathy. J Ophthalmol. 2019; 2019: 2648267.

Doi: $10.1155 / 2019 / 2648267$.

2. Dong N, Xu B, Wang B. Aqueous cytokines as predictors of macular edema in patients with diabetes following uncomplicated phacoemulsification cataract surgery. Bio Med Research International. 2015; 2015: 8. Doi: 10.1155/2015/126984.126984 -

3. Lim LL, Morrison JL, Constantinou M, Rogers S, Sandhu SS, Wickremasinghe SS, et al. Diabetic macular edema at the time of cataract surgery trial: a prospective, randomized clinical trial of intravitreous bevacizumab versus triamcinolone in patients with diabetic macular oedema at the time of cataract surgerypreliminary 6 month results. Clin Exp Ophthalmol. 2016; 44 (4): 233-242. Doi: 10.1111/ceo.12720.

4. Virgili G, Parravano M, Evans JR, Gordon I, Lucenteforte E. Anti-vascular endothelial growth factor for diabetic macular oedema: a network metaanalysis. Cochrane Database Syst Rev. 2017; 6.

Doi: 10.1002/14651858.CD007419.pub5.CD007419

5. Lin WH, Lu M, Tang HY, Zeng ZR. Clinical application of Ranibizumab in the therapy of diabetic cataract. Guoji Yanke Zazhi. 2015; 15: 880-882.
6. Cheng MZ, Wang C, Chen M. Phacoemulsification combined with intravitreal injection of lucentis for diabetic cataract. Recent Adv Ophthalmol. 2016; 36: 754-756.

7. Bakri SJ, Snyder MR, Reid JM, Pulido JS, Singh RJ. Pharmacokinetics of intravitreal bevacizumab (Avastin). Ophthalmology, 2007; 114: 855-849.

8. Li T. The effect of senile cataract surgery combined with vitreous cavity injection of monoclonal antibody on diabetic macular edema. China Cont Med Edu. 2016; 8: 149-151

9. Audren F, Lecleire-Collet A, Erginay A, Haouchine B, Benosman R, Bergmann JF, et al. Intravitreal triamcinolone acetonide for diffuse diabetic macular edema: phase 2 trial comparing $4 \mathrm{mg}$ vs. $2 \mathrm{mg}$. Am J Ophthalmol. 2006; 142: 794-799.

10. Haritoglou C, Kook D, Neubauer A, Wolf A, Priglinger S, Strauss R, et al. Intravitreal bevacizumab (Avastin) therapy for persistent diffuse diabetic macular edema. Retina. 2006; 26: 999-1005.

11. Scott IU, Edwards AR, Beck RW. A phase II randomized clinical trial of intravitreal bevacizumab for diabetic macular edema. Ophthalmology, 2007; 14: 1860-1867.

12. Takamura Y, Kubo E, Akagi Y. Analysis of the effect of intravitreal Bevacizumab injection on diabetic macular edema after cataract surgery. Ophthalmology, 2009; 116 (6): 1151-1157.

13. Lanzagorta-Aresti A, Palacios-Pozo E, Menezo Rozalen JL, Naveatejerina A. Prevention of vision loss after cataract surgery in diabetic macular edema with intravitreal bevacizumab. Retina, 2009; 29 (4): 530-535

14. Cheema RA, Al-Mubarak MM, Amin YM, Cheema MA. Role of combined cataract surgery and intravitreal bevacizumab injection in preventing progression of diabetic retinopathy. J Cat Refract Surg. 2009; 35 (1): 18-25. Doi: 10.1016/j.jcrs.2008.09.019.

15. Chae JB, Joe SG, Yang SJ. Effect of combined cataract surgery and Ranibizumab injection in postoperative macular edema in non-proliferative diabetic retinopathy. Retina, 2014; 34 (1): 149-156. Doi: 10.1097/iae.0b013e3182979b9e.

16. Lin WH, Lu M, Tang HY, Zeng ZR. Clinical application of Ranibizumab in the therapy of diabetic cataract. Guoji Yanke Zazhi. 2015; 15: 880-882.

17. Sze AM, Luk FO, Yip TP, Lee GK, Chan CK. Use of intravitreal dexamethasone implant in patients with cataract and macular edema undergoing phacoemulsification. Eur J Ophthalmol. 2015; 25 (2): 168-172. Doi: 10.5301/ejo.5000523.

18. Elman MJ, Qin H, Aiello LP, Beck RW, Bressler NM, Ferris FL, et al. Intravitreal ranibizumab for diabetic macular edema with prompt versus deferred laser treatment: three-year randomized trial results. Ophthalmology, 2012; 119: 2312-2318. 
19. Hartnett ME, Tinkham N, Paynter L. Aqueous vascular endothelial growth factor as a predictor of macular thickening following cataract surgery in patients with diabetes mellitus. Am J Ophthalmol. 2009; 148 (6): 895-901.

20. Fard MA, Abyane YA, Malihi M. Prophylactic intravitreal bevacizumab for diabetic macular edema (thickening) after cataract surgery: prospective randomized study. Eur J Ophthalmol. 2011; 21 (3): 276-281.

21. Cetin EN, Yildirim C. Adjuvant treatment modalities to control macular edema in diabetic patients undergoing cataract surgery. Int Ophthalmol. 2013; 33 (5): 605-610.

\section{Authors' Designation and Contribution}

Ali Afzal Bodla; Associate Professor: Concepts, Design, Manuscript editing, Manuscript review.

Syeda Minahil Kazmi; Consultant Ophthalmologist: Data acquisition, Data analysis, Statistical analysis, Manuscript review.

Noor Tariq; Consultant Ophthalmologist: Literature search, Data analysis, Manuscript preparation, Manuscript editing.

Ayema Moazzam; Consultant Ophthalmologist: Data acquisition, Statistical analysis, Manuscript preparation, Manuscript editing.

Muhammad Muneeb Aman; Consultant Ophthalmologist: Literature search, Data acquisition, Data analysis, Statistical analysis.

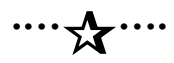

